\title{
Resíduo Sólido Orgânico em Unidade de Alimentação e Nutrição Hospitalar: a Contribuição do Resto dos Pacientes
}

\author{
Tânia Regina Kinasz ${ }^{1}$, Ana Laura Almeida Dias², \\ Dálete Campos Mariano ${ }^{3}$, Mayara Barbosa Lima ${ }^{4}$
}

\begin{abstract}
RESUMO
Neste texto avaliou-se a geração de resíduo sólido orgânico advindo do resto de pacientes de acordo com o tipo de dieta e a clínica de internação, e os fatores que influenciaram nessa geração. Realizou-se um estudo transversal descritivo num hospital universitário localizado no município de Cuiabá/MT. As amostras foram coletadas durante o jantar. Para o cálculo da porção média de refeição distribuída, per capita médio e índice médio do resto, foi utilizado o método de pesagem, e como padrão de referência o índice inferior a $20 \%$. Enquetes de satisfação realizadas com 41 pacientes avaliaram os fatores que influenciam nessa geração. Observou-se que a geração variou de acordo com a consistência das dietas e clínicas de internação. Hábitos e preferências alimentares, sensação de plenitude gástrica, porção elevada de refeição, náusea, inapetência e o horário do jantar, foram os motivos mais citados para justificar a geração. Concluiu-se que a redefinição dos per capita dos alimentos, de acordo com as preparações, redefinição das porções conforme a consistência da dieta por clínica de internação, padronização dos utensílios e o cumprimento desta padronização, poderão contribuir para a diminuição dos índices de resíduo orgânico advindo do resto.
\end{abstract}

Palavras-chave: Resíduo sólido orgânico. Resto. Unidade de alimentação e nutrição hospitalar.

SOLID ORGANIC WASTE IN FOODSERVICES HOSPITAL: THE CONTRIBUTION OF THE PLATE WASTE PATIENTS

ABSTRACT

The objective was to analyze the generation of solid organic waste originating from plate waste by patients the as internment clinic and the type of diet of a university hospital in the Cuiabá city of Mato Grosso, Brazil, and the factors that had contributed in this generation. An descriptive transversal study was carried. Were analyzed. 41 patients were questioned. For the calculation of the average portion meal of distributed and of the portion meal of plate waste the weight, method was used and as reference standard the rate of $20 \%$ for the plate waste. The index was determined by type of diet. For evaluation of the factors that, in the vision of the patients influence in the generation of solid organic waste happened of the plate waste ingestion questionnaire with open question was used. It was observed that the generation of solid organic waste originating of plate waste varied according to the consistency of the diets and clinics internment. Alimentary habits and preferences, sensation of gastric fullness, high portion, nauseous, inappetence, dinner time had been the cited reasons more for the rejection of the diet. The redefinition of per capita in accordance with the preparations, the redefinition of portions in accordance with the consistency of the diet for clinic internment and the standardization of the utensils and the execution of this standardization will be able to contribute for the reduction of the rate of organic waste originating the food wastage.

Keywords: Solid organic waste. Plate waste. Hospital food and nutrition services.

RECEBIDO EM: $14 / 1 / 2019$

MODIFICAÇÕES REQUERIDAS EM: 28/8/2019

ACEITO EM: 8/9/2019

\footnotetext{
Nutricionista. Doutora em Ciências pela Universidade Federal de São Paulo. Professora Associada do Departamento de Alimentos e Nutrição da Faculdade de Nutrição da Universidade Federal de Mato Grosso - Campus Universitário Cuiabá, Cuiabá/MT, Brasil. trkinasz@hotmail.com

${ }^{2}$ Nutricionista graduada pela Faculdade de Nutrição da Universidade Federal de Mato Grosso - Campus Universitário Cuiabá, Cuiabá/MT, Brasil. analaura. almdias@gmail.com

${ }^{3}$ Nutricionista graduada pela Faculdade de Nutrição da Universidade Federal de Mato Grosso - Campus Universitário Cuiabá, Cuiabá/MT, Brasil. daletemariano@yahoo.com.br

${ }^{4}$ Nutricionista graduada pela Faculdade de Nutrição da Universidade Federal de Mato Grosso - Campus Universitário Cuiabá, Cuiabá/MT, Brasil.
} 


\section{INTRODUÇÃO}

O objetivo da Unidade de Alimentação e Nutrição (UAN) hospitalar é o de fornecer alimentação equilibrada à clientela sadia e enferma, e, no caso da população enferma, busca recuperar e conservar a saúde (MEZOMO, 1989).

Para atender a esses objetivos, as dietas hospitalares são padronizadas seguindo as modificações qualitativas e quantitativas da alimentação normal, assim como da consistência, temperatura, volume, valor calórico total, alterações de macronutrientes e restrições de nutrientes, podendo ser classificadas a partir das suas principais características, indicações e alimentos ou preparações a serem servidas (MACULEVICIUS; DIAS, 2002; ISOSAKI; CARDOSO; OLIVEIRA, 2009).

É de responsabilidade do nutricionista a garantia da produção e distribuição de refeições sob os aspectos de quantidade, qualidade e segurança sanitária, e, no caso da clientela enferma, a prestação de assistência nutricional e dietoterápica (BRASIL, 2018).

Especificamente quanto à Unidade de Alimentação e Nutrição (UAN) hospitalar, pesquisas demonstraram, entre outros aspectos, a importância do cuidado e da valorização nutricional das refeições oferecidas aos pacientes (GARCIA, 2006; DE SETA et al., 2010), da ingestão insuficiente de alimentos (DUPERTUIS et al., 2003; BARTON et al., 2000), da oferta de alimentos seguros (SOUZA; CAMPOS, 2003), da aceitação da alimentação (WATTERS et al., 2003; WRIGHT; CONELLY; CAPRA, 2006; YABUTA; CARDOSO; ISOSAKI, 2006), da redução do desperdício de alimentos e dos custos (FOLIO; SULLIVAN-MAILLET; TOUGER-DECKER, 2002; CONNORS; ROZELL, 2004; NONINO-BORGES et al., 2006).

A responsabilidade técnica do nutricionista impõe novas perspectivas de gerenciamento cuja importância vai além destes aspectos e quando o aspecto socioambiental se faz presente, uma vez que a utilização racional dos recursos naturais e processos ambientalmente adequados são necessários (KINASZ; WERLE, 2006; KINASZ et al., 2010), com a observância dos aspectos de legislação.

A Lei no 12.305/2010 (BRASIL, 2010) instituiu a Política Nacional de Resíduos Sólidos, apontando as diretrizes relativas à gestão integrada e ao gerenciamento de resíduos sólidos, às responsabilidades dos geradores no gerenciamento e à destinação adequada destes resíduos, com obrigatoriedade da elaboração do Plano de Gerenciamento de Resíduos Sólidos para alguns geradores.
Entre os geradores que estão sujeitos à elaboração deste plano encontram-se os serviços de saúde, que devem observar a Resolução RDC no 306/2004 (BRASIL, 2004), que dispõe sobre o regulamento técnico para o gerenciamento de resíduos de serviços de saúde, e a RDC no 358/2005 (BRASIL, 2005), que dispõe sobre o tratamento e a disposição final dos resíduos dos serviços de saúde.

A geração de resíduos em unidades de saúde depende, entre outros fatores, do tipo da unidade, complexidade dos procedimentos, do tamanho da unidade (SILVA et al., 2005) e da alimentação oferecida.

Dados de composição gravimétrica dos resíduos de serviços de saúde demonstram que as UANs inseridas nestes serviços são responsáveis por uma geração variável de 45\% (SOARES; CASTILHOS; MACEDO, 1997) a 21\% (NAGASHIMA; BARROS; FONTES, 2007) e, segundo esses autores, destes índices $65 \%$ a $47 \%$ são considerados resíduos comuns, nos quais se incluem os resíduos orgânicos.

No Brasil, a abordagem sobre a geração de resíduos orgânicos e os impactos ambientais foi incorporada recentemente em diferentes modalidades de UANs que atendem a coletividades sadias. Há, entretanto, carência de estudos que avaliem a geração destes resíduos em UAN que atende a coletividade enferma.

Pesquisas realizadas no Brasil em UANs que atendem a trabalhadores, demonstraram que a maior fonte de geração de resíduos orgânicos se dá na etapa da distribuição de refeições, por meio do resto (KINASZ; WERLE, 2006, SPINELLI; CALE, 2009, KINASZ et al., 2010; CHAMBERLEM; KINASZ; CAMPOS, 2012), configurando-se em desperdício de alimentos.

Há, todavia, há carência de estudos sobre essa temática em pacientes hospitalizados. Assim, avaliar a geração de resíduo sólido orgânico advindo do resto de pacientes de acordo com o tipo de dieta e a clínica de internação, e os fatores que influenciaram nessa geração, além de fornecer elementos para análise da adequação da dieta, possibilita a tomada de medidas que podem contribuir com a minimização na geração desses resíduos e o desperdício de alimentos, fornecendo subsídios para a eficiência na gestão da UAN como também para elaboração e estabelecimento do Plano de Gerenciamento de Resíduos Sólidos da instituição hospitalar à qual está inserida.

\section{MÉTODO}

Trata-se de um estudo de caso, transversal, descritivo, realizado em um hospital universitário público, localizado no município de Cuiabá/MT. 
Foram selecionados pacientes de ambos os sexos, com mais de dois e menos de sete dias de internação que, após diagnóstico médico, tiveram a prescrição de dieta via oral de consistência normal, branda ou pastosa especial, e que consentiram na realização do estudo.

Foram excluídos os pacientes com prescrição de dieta hipolipídica e/ou hipossódica, independente das consistências, uma vez que supostamente haveria maior probabilidade de recusa da refeição, influenciando na geração de resíduos orgânicos. Os pacientes internados na pediatria também foram excluídos por terem refeições com porcionamento diferenciado.

As amostras das refeições foram coletadas no jantar, de segunda à sexta-feira, no período de setembro a dezembro de 2010, totalizando 184 dietas, sendo 12 na clínica médica, 28 na clínica cirúrgica e 144 da clínica de ginecologia e obstetrícia.

Para o cálculo da porção média de refeição distribuída, todas as preparações que compunham o cardápio foram diariamente porcionadas de acordo com a padronização estipulada e consistência da dieta, e pesadas descontando-se o peso estimado dos ossos e o peso das legumeiras, sendo estas etiquetadas na parte inferior para posterior identificação e pesagem do resto.

O per capita médio de resto foi obtido a partir da pesagem diária do resto de cada preparação, descontando-se o peso dos recipientes e dos ossos.

Para todas as pesagens foi utilizada uma balança digital de precisão, observando-se a regulagem, existência de vistoria e lacre do Instituto Nacional de Metrologia, Normalização e Qualidade Industrial - Inmetro.

Calculou-se o índice de resto multiplicando-se por cem o quociente entre o per capita médio de resto e a porção média de refeição distribuída. Utilizou-se como padrão de referência índice inferior a $20 \%$ preconizado teoricamente por Mezomo (1989).

O método escolhido para avaliar os fatores que, na visão dos pacientes influenciam na geração de resíduos sólidos orgânicos advindos do resto, foi a pesquisa descritiva utilizando-se um questionário com uma única pergunta aberta sobre o motivo da não aceitação, parcial ou total, da dieta oferecida. O questionário foi aplicado a 41 pacientes que se disponibilizaram a responde-lo.

O estudo foi aprovado pelo Comitê de Ética em Pesquisa do Hospital Universitário Júlio Muller, da Universidade Federal de Mato Grosso, sob o número 946/CEP-HUJM/2010.
Os dados foram organizados por meio da utilização do banco de dados do Microsoft ${ }^{\circledR}$ Excel 2007, e para a análise utilizou-se o programa SPSS ${ }^{\circledR} 17.0$, sendo apresentados em tabelas com distribuição simples e relativa.

\section{RESULTADOS E DISCUSSÕES}

\section{Caracterização da Unidade, Tipos de Dietas e Tipo do Cardápio}

A UAN é do tipo autogestão, tendo em seu quadro três nutricionistas no Serviço de Produção de Refeições e sete no Serviço de Nutrição Clínica, responsáveis pelo planejamento, organização, coordenação, comando, controle e avaliação das atividades realizadas, bem como pela assistência e educação nutricional da coletividade sadia e enferma.

A produção e a distribuição das dietas são centralizadas, sendo padronizadas e porcionadas em legumeiras em aço inox, com divisão das preparações, e transportadas até as clínicas de internação por meio de carros de distribuição.

O cardápio servido é categorizado como simples, composto por entrada, prato principal, guarnição, acompanhamento e sobremesa. A sobremesa não foi avaliada, pois a maioria dos pacientes a consumiam após o horário do jantar.

As dietas avaliadas foram as de consistência normal, branda e pastosa especial, sendo todas de característica normoglicídica, normolipídica e normoproteica. A dieta pastosa não foi avaliada porque durante o estudo houve prescrição desta apenas na clínica cirúrgica.

A dieta normal é indicada para indivíduos que não necessitam de modificação em nutrientes e consistência, não havendo restrições de alimentos, preparações, especiarias e condimentos (CARUSO; SIMONY; SILVA, 2004). A composição calórica recomendada é de 1.800 a $2.500 \mathrm{kcal} / \mathrm{dia}$, com fracionamento de 5 a 6 refeições/dia (SIMON et al., 2014)

A dieta branda é indicada para indivíduos com problemas mecânicos de mastigação, deglutição e digestão que impedem o consumo da dieta normal, sendo necessário o abrandamento do tecido conectivo e da celulose dos alimentos pela cocção ou ação mecânica. Restringe-se especiarias e condimentos picantes, frituras, hortaliças cruas, embutidos e conservas (CARUSO; SIMONY; SILVA, 2004). A composição calórica recomendada é de 1.800 a $2.200 \mathrm{kcal} / \mathrm{dia}$, com fracionamento de 5 a 6 refeições/dia (SIMON et al., 2014). 
A dieta pastosa é indicada para indivíduos com problemas mecânicos de mastigação, deglutição e distúrbios motores. Os alimentos devem estar em forma de purês ou cremes e mingaus; as carnes moídas, desfiadas ou liquidificadas, arroz papa, massas bem-cozidas, caldos de leguminosas, pães e frutas macias. Restringe-se alimentos "duros", carnes inteiras e grãos (SIMON et al., 2014). A composição calórica recomendada é de 1.800 a $2.200 \mathrm{kcal} / \mathrm{dia}$, com fracionamento de 5 a 6 refeições/dia (SIMON et al., 2014).

A dieta pastosa especial foi desenvolvida pelos nutricionistas do serviço para atender aos pacientes sem restrições a determinadas preparações da dieta branda e/ou pastosa, restringindo-se especiarias e condimentos picantes, frituras, hortaliças cruas, embutidos e conservas. As carnes são oferecidas moídas ou desfiadas, o arroz em consistência de papa, o feijão liquidificado e os legumes refogados ou cozidos.

\section{Análise da Porção Média de Refeição, do Per Capita Médio e do Índice Médio do Resto}

Observou-se que houve variação na quantidade da porção de refeição servida, nos per capita e nos índices de resto para a mesma consistência da dieta, entre as consistências das dietas e em todas as clínicas de internação (Tabela 1).

Utilizando-se como referência o índice de até $20 \%$ de resto, observou-se que a dieta normal teve índice superior na clinica cirúrgica, a dieta branda em todas as clínicas de internação e a dieta pastosa especial nas clínicas médica e cirúrgica. Em contrapartida, foi observado índice inferior para a dieta normal na clínica médica e de ginecologia e obstetrícia para a dieta pastosa especial na clínica de ginecologia e obstetrícia.
Fatores que Contribuíram com a Rejeição das Dietas Oferecidas Segundo a Visão dos Pacientes

Hábitos e preferências alimentares, sensação de plenitude gástrica, porção elevada de refeição, náusea, inapetência e horário do jantar, foram os motivos mais citados para a rejeição da dieta. Os fatores como temperatura da refeição, qualidade higiênica da refeição, tabu alimentar, desconforto causado por cateter, estresse pela internação e horário da amamentação, foram os motivos menos citados (Tabela 2).

Tabela 2 - Fatores que contribuíram com a rejeição das dietas oferecidas nas clínicas Médica, Cirúrgica e de Ginecologia e Obstetrícia, segundo a visão dos pacientes

\begin{tabular}{l|c|c}
\hline \multicolumn{1}{c|}{ Fatores de rejeição da dieta } & $\mathbf{n}$ & $\mathbf{\%}$ \\
\hline Hábitos e preferências alimentares & 14 & 34,1 \\
Sensação de plenitude gástrica & 5 & 12,2 \\
Porção elevada de refeição & 5 & 12,2 \\
Náusea & 4 & 9,7 \\
Inapetência & 4 & 9,7 \\
Horário do jantar & 3 & 7,3 \\
Temperatura da refeição & 1 & 2,4 \\
Qualidade higiênica da refeição & 1 & 2,4 \\
Tabu alimentar & 1 & 2,4 \\
Desconforto causado por cateter & 1 & 2,4 \\
Estresse pela internação & 1 & 2,4 \\
Horário da amamentação & 1 & 2,4 \\
\hline Total & $\mathbf{4 1}$ & $\mathbf{1 0 0}$ \\
\hline
\end{tabular}

Barton et al. (2000) realizaram um estudo em um hospital universitário no Reino Unido e obtiveram índice aproximado de $40 \%$ para resto resultante da subavaliação do estado nutricional e da capacidade de ingestão alimentar dos pacientes. Nonino-Borges et al. (2006), em estudo efetuado em uma unidade

Tabela 1 - Porção média de refeição (g), per capita médio de resto (g) e índice médio de resto (\%), segundo a consistência das dietas e clínica de internação

\begin{tabular}{c|c|c|c|c|c|c}
\hline Clínica de Internação & \multicolumn{2}{|c|}{ Dieta Normal } & \multicolumn{2}{c|}{ Dieta Branda } & \multicolumn{2}{c}{ Dieta Pastosa Especial } \\
\hline & Porção & Per capita resto & Porção & Per capita resto & Porção & Per capita resto \\
\hline Clínica Médica & 444,9 & $\begin{array}{c}17,5 \\
(3,9 \%)\end{array}$ & 527,7 & $\begin{array}{c}111,0 \\
(21,0 \%)\end{array}$ & 428,9 & $\begin{array}{c}133,9 \\
(31,2 \%)\end{array}$ \\
\hline Clínica Cirúrgica & 425,4 & $\begin{array}{c}107,7 \\
(25,3 \%)\end{array}$ & 491,3 & $\begin{array}{c}116,1 \\
(23,6 \%)\end{array}$ & 392,0 & $\begin{array}{c}78,0 \\
(20,0 \%)\end{array}$ \\
\hline $\begin{array}{c}\text { Clínica de Ginecologia e } \\
\text { Obstetrícia }\end{array}$ & 452,7 & 80,9 & 469,3 & 127,8 & 432,9 & 31,5 \\
& & $(17,9 \%)$ & & $(27,2 \%)$ & & $(7,3 \%)$ \\
\hline
\end{tabular}


hospitalar brasileira, obtiveram índice médio de $27 \%$ no almoço e $25 \%$ no jantar oferecidos aos pacientes, revelando perdas financeiras importantes. Índices menores foram observados no Brasil por Contri et al. (2010), variando de $12 \%$ a $15 \%$.

Mezomo (1989) teoricamente preconiza como padrão de referência o índice de até $20 \%$ para coletividade enferma, sendo este índice utilizado em muitos estudos brasileiros. Platt, Eddy e Pellett (1963), entretanto, estudando a coletividade enferma, há muito reportaram que a utilização de índice de $10 \%$ era excessiva.

Diante da constatação de que a questão não se resume apenas à aceitabilidade da dieta e à possibilidade de ocorrência de deficiências nutricionais dos pacientes, mas na geração de resíduos como fruto do desperdício de alimentos, é imperioso sugerir índice inferior a $10 \%$ como padrão de referência. Para o caso de pacientes em que os fatores relacionados à doença interferem demasiadamente na aceitabilidade, condutas particularizadas, como fracionamento, consistência e preferências alimentares, devem ser avaliadas.

Estudos com pacientes hospitalizados demonstraram que o aspecto visual (HIRSCH et al., 1997; SHAHAR et al., 2002, CONTRI et al., 2010), consistência (CONTRI et al., 2010), sabor (SHAHAR et al., 2002, WRIGHT; CONELLY; CAPRA, 2006), bem como variedade, odor e textura (WHIGHT; CONELLY; CAPRA, 2006), estão relacionados à satisfação com a dieta recebida e influenciam seu consumo. Ações que contemplem a adequação destes aspectos minimizariam a geração de resíduos orgânicos. Assim, pesquisas para verificação desta relação seriam pertinentes.

Poulain (2004) e Sousa e Proença (2005) destacaram a importância da função terapêutica da alimentação na internação, com a necessidade da contemplação dos aspectos sensoriais e simbólicos. Estudo realizado por Kelly (1999) reforça a importância dos aspectos sensoriais na aceitabilidade da dieta.

Neste estudo, observou-se que na dieta normal houve variação dos alimentos utilizados nas preparações servidas, como entrada, prato proteico e guarnição, bem como variação de textura, cor e no tipo de calor utilizado no preparo. Nas demais dietas, observou-se monotonia nos tipos de alimentos e de preparações servidas, como entrada, sendo a sopa a mais utilizada. A consistência, os tipos de alimentos e o modo de preparo recomendados para as demais consistências das dietas, também podem ter acarretado a recusa da refeição, contribuindo para a geração de resíduo orgânico.
Sendo assim, no planejamento das dietas a observância dos aspectos sensoriais e simbólicos, como preconizado por Poulain (2004) e Sousa e Proença (2005), assim como a orientação do paciente e o acompanhamento da aceitabilidade da dieta, são imprescindíveis não somente sob o aspecto de atendimento das necessidades nutricionais dos pacientes.

É sabido que limitações impostas pelas doenças influenciam na aceitabilidade das dietas, assim como outros fatores. Allison (2003), ao avaliar pacientes hospitalizados, relatou que o ambiente de alimentação, o tamanho da porção, a necessidade de ajuda e de utensílios especiais para se alimentar, interferem no consumo alimentar. Dilly e Shanklin (1998) descreveram que o tipo de preparação e o sistema de distribuição das refeições podem influenciar no desperdício de alimentos dos pacientes.

Para que o porcionamento seja adequado às necessidades do paciente, é necessária a qualificação dos funcionários e a padronização de utensílios para cada tipo de preparação.

Neste estudo foi observado que, embora houvesse utensílios padronizados e o relato do nutricionista responsável pelo serviço sobre realização de treinamentos teóricos e práticos com frequência de seis meses a um ano, os funcionários não cumpriam efetivamente o que era preconizado. Isso pode explicar a variação no porcionamento das refeições para uma mesma consistência entre as clínicas de internação (Tabela 1). Por outro lado, o tipo de distribuição utilizado deixa ao paciente apenas a opção de aceitar, total ou parcialmente, ou até mesmo rejeitar a preparação por impossibilidade de escolha destas e/ou opinar sobre a quantidade servida.

Além da constatação da porção elevada de refeição, o horário de distribuição da mesma (Tabela 2) merece atenção na medida em que a unidade oferece seis refeições: desjejum, colação, almoço, merenda, jantar e ceia, que, muitas vezes, não são realizadas fora do ambiente hospitalar. O intervalo entre as refeições, aliado ao tamanho da porção, pode ter contribuído. Outros estudos para verificação desta possível relação são pertinentes.

Índices elevados de resto vão além dos aspectos ligados à geração de resíduos orgânicos, uma vez que podem contribuir para a ocorrência de deficiências nutricionais dos pacientes, como demonstrou o estudo de Barton et al. (2000), ao constatar que o índice de rejeição de $40 \%$ resultou em aporte energético e proteico inferior a $80 \%$ das recomendações. 
Ressalta-se que na atuação do nutricionista (BRASIL, 2018), além da garantia da produção e distribuição de refeições sob os aspectos de quantidade, qualidade, segurança sanitária, custo e satisfação da clientela atendida, deva-se ainda se atentar quanto à responsabilidade socioambiental, uma vez que é responsável pelo gerenciamento dos resíduos sólidos gerados nas UANs, quando ações de minimização na geração, reutilização e reciclagem dos resíduos passíveis desta destinação e ações de educação ambiental se fazem necessárias.

\section{CONCLUSÕES}

Observou-se que a dieta normal teve índice superior ao fixado como aceitável na clínica cirúrgica, a dieta branda teve índices superiores em todas as clínicas de internação e a dieta pastosa especial nas clínicas médica e cirúrgica.

Também foi observado índice inferior ao fixado como aceitável para a dieta normal na clínica médica e para a dieta pastosa especial na clínica de ginecologia e obstetrícia.

A redefinição dos per capita dos alimentos de acordo com as preparações, a redefinição das porções conforme a consistência da dieta por clínica de internação, a padronização dos utensílios e o cumprimento desta padronização, a oferta de preparações que atendam aos hábitos e preferências alimentares e a adequação do horário do jantar, poderão contribuir com o aumento da satisfação pela dieta e, consequentemente, com a diminuição dos índices de resíduos orgânicos advindos do resto.

Recomenda-se a revisão da utilização do padrão de $20 \%$ para índice de resto de população enferma, preconizado teoricamente na literatura.

Sugere-se, como padrão de referência para índice de resto de população enferma, taxa inferior a $10 \%$.

Outros estudos são necessários para verificar a importância relativa dos diferentes fatores de rejeição da dieta.

\section{REFERÊNCIAS}

ALLISON, S. P. Hospital food and treatment. Clin. Nutr., v. 22, n. 2, p. 13-14, 2003.

BARTON, A. D. et al. High food wastage and low nutritional intakes in hospital patients. Clin. Nutr., v. 19, n. 6, p. 445449, 2000.
BRASIL. Agência Nacional de Vigilância Sanitária. Resolução RDC no 306, de 7 de dezembro de 2004. Dispõe sobre o regulamento técnico para o gerenciamento de resíduos de serviços de saúde. Diário Oficial da União, Poder Executivo, Brasília, DF, 10 dez. 2004.

BRASIL. Conselho Federal de Nutricionistas Resolução no $600 / 2018$ de 20 de abr. 2018. Dispõe sobre a definição das áreas de atuação do nutricionista e suas atribuições, indica parâmetros numéricos mínimos de referência, por área de atuação, para a efetividade dos serviços prestados à sociedade e dá outras providências. Diário Oficial da União [Internet]. 2018 abr. Disponível em: http://www.cfn.org.br/ legislação/ resolução.htm. Acesso em: 30 ago. 2019.

BRASIL. Conselho Nacional de Meio Ambiente. Resolução RDC no 358 de 29 de abril de 2005. Dispõe sobre o tratamento e a disposição final dos resíduos dos serviços de saúde e dá outras providências. Diário Oficial da União, Poder Executivo, Brasília, DF, 4 maio 2005.

BRASIL. Lei no 12.305/2010. Institui a Política Nacional de Resíduos Sólidos; altera a Lei no 9.605, de 12 de fevereiro de 1998 e dá outras providências. Diário Oficial [da] República Federativa do Brasil, Brasília, DF, 2 de agosto de 2010. Disponível em: http://www.planalto.gov.br/ccivil_03/_ ato2007-2010/2010/lei/l12305.htm.

CARUSO, L.; SIMONY, R. F.; SILVA, A. L. N. D. Dietas hospitalares: uma abordagem na prática clínica. São Paulo: Atheneu, 2004. 148p.

CHAMBERLEM, S. R.; KINASZ, T. R.; CAMPOS, M. P. F. F. Resto de ingestão e sobra descartada - fonte de geração de resíduos orgânicos em Unidades de Alimentação e Nutrição em Cuiabá - MT. Alimentação e Nutrição, Araraquara, v. 23, n. 2, abr. 2012. Disponível em: http://serv-bib.fcfar.unesp.br/ seer/index.php/alimentos/article/view/2039/2039. Acesso em: 25 set. 2017.

CHIARELLO, P. G.; SANTOS, J. S.; MARCHINI, J. S. Desperdício de alimentos intra-hospitalar. Revista Nutrição, Campinas, v. 19, n. 3, p. 349-356, 2006.

CONNORS, P.; ROZELL, S. Using visual plate waste study to monitor menu performance. J Am Diet Assoc., n. 104, p. 9496, 2004.

CONTRI, P. V. et al. Porcionamento e consumo de saladas por mulheres com dieta geral em unidade de internação hospitalar. Alimentos e Nutrição, Araraquara, v. 21, n. 1, p. 141-147, 2010.

DE SETA, M. H. et al. Cuidado nutricional em hospitais públicos de quatro Estados brasileiros: contribuições da avaliação em saúde à vigilância sanitária de serviços. Ciências \& Saúde Coletiva, Rio de Janeiro, v. 15, n. 3, p. 3.413-3.422, 2010.

DILLY, G.; SHANKLIN, C. Characterisation of waste in two military hospital foodservice operations. Journal American Dietetic Association, v. 98, n. 9, p. 357-360, 1998.

DUPERTUIS, Y. M. et al. Food intake in 1707 hospitalised patients: a prospective comprehensive hospital survey. Clinical Nutrition, v. 22, n. 2, p. 115-123, 2003. 
FOLIO, D.; SULLIVAN-MAILLET, J.; TOUGER-DECKER, R. The spoken menu concept of patient foodservice delivery systems increase overall patient satisfaction, therapeutic and tray accuracy, and cost is neutral for food and labor. J. Am. Diet. Assoc., v. 102, p. 546-548, 2002.

GARCIA, R. W. D. A dieta hospitalar na perspectiva dos sujeitos envolvidos em sua produção e em seu planejamento. Revista de Nutrição, Campinas, v. 19, n. 2, p. 129-144, 2006.

HIRSCH, K. M. et al. Factors influencing plate waste by hospitalized patient. J Am Dietet Assoc., v. 75, n. 3, p. 270-273, 1997.

ISOSAKI, M.; CARDOSO, E.; OLIVEIRA, A. De. Manual de dietoterapia e avaliação nutricional: Serviço de Nutrição e dietética do Instituto do Coração - HCFMUSP. 2. ed. São Paulo: Editora Atheneu, 2009.

KELLY, L. Audit of food wastage: differences between a plated and bulk system of meal provision. Journal of Human Nutrition and Dietetics, v. 12, p. 415-424, 1999.

KINASZ, T. R. et al. Resíduos sólidos orgânicos em unidades de alimentação e nutrição: estudo de fatores relevantes na geração de resto, sobras descartadas e de desperdício no preparo de hortaliças e frutas. In: KINASZ, T. R. Resíduos sólidos orgânicos em unidades de alimentação e nutrição: estudo de fatores relevantes na geração de resto, de sobras descartadas e no desperdício do preparo de hortaliças e frutas. 2010. Tese (Doutorado) -Universidade Federal de São Paulo, São Paulo, 2010.

KINASZ, T. R.; WERLE, H. J. S. Composição física, origem e fluxo da produção de resíduos sólidos nos serviços de alimentação e nutrição do aglomerado urbano Cuiabá - Várzea Grande, MT: questões ambientais. Revista Higiene Alimentar, Mirandópolis, v. 20, n. 144, p. 64-71, set. 2006.

MACULEVICIUS, J.; DIAS, M. C. G. Dietas orais hospitalares. In: WAITZBERG, D. L. Nutrição oral, enteral e parenteral na prática clínica. 3. ed. São Paulo: Editora Atheneu, 2002, p. 465-479. V. 1. Cap. 30.

MEZOMO. I. F. B. Serviços de nutrição: administração e organização. São Paulo: Cedas, 1989. 440p.

NAGASHIMA, L. A.; BARROS, J. R. C.; FONTES, C. E. R. Análise da produção e taxa de geração de resíduos sólidos de serviço de saúde do hospital universitário regional de Maringá. Acta Sci. Technol., Maringá, v. 29, n. 2, p. 131-139, 2007.

NONINO-BORGES, C. B. et al. Desperdício de alimentos intra-hospitalar. Nutrição, Campinas, SP, v. 19, n. 3, p. 349356, 2006.

PLATT, B. S.; EDDY, T. P.; PELLETT, P. L. Food in Hospitals. London: Oxford University Press, 1963.
POULAIN, J. P. Sociologias da alimentação: os comedores e o espaço social alimentar. Florianópolis: Editora da UFSC, 2004.

SCOTTON, V.; KINAZ, T. R.; COELHO, S. R. M. Desperdícios de alimentos em Unidades de Alimentação e Nutrição: A contribuição do resto ingestão e da sobra. Revista Higiene Alimentar, Mirandópolis, v. 24, n. 186/187, p. 19-24, 2010.

SHAHAR, S. et al. Food intakes and preferences of hospitalised geriatric patients. BMC Geriatrics, v. 2, n. 1, 2002. Available from: http://www.biomedcentral.com/14712318/2/3. Cited: 23 Nov. 2017.

SILVA, C. E. et al. Medical wastes management in the south of Brazil. Waste Management, v. 25, p. 600-605, 2005.

SIMON, M. I. S. S. et al. Manual de dietas hospitalares. São Paulo: Editora Atheneu, 2014. 82p.

SOARES, I. C. C. et al. Quantificação e análise do custo da sobra limpa em unidades de alimentação e nutrição de uma empresa de grande porte. Revista de Nutrição, Campinas, v. 24, n. 4, p. 593-604, 2011.

SOARES, R. S.; CASTILHOS JR., A. B.; MACEDO, M. C. Diagnóstico da produção de resíduos de serviços de saúde estudo de caso: Hospital Universitário de Florianópolis - SC. In: ASSOCIAÇÃO BRASILEIRA DE ENGENHARIA SANITÁRIA E AMBIENTAL. Trabalhos técnicos. Rio de Janeiro: Abes, 1997. p. 7.

SOUSA, A. A.; PROENÇA, R. P. C. La gestion des soins nutritionnels dans le secteur hospitalier: une etude comparative Bresil-France. Rech Soins Infirm., n. 83, p. 28-33, 2005.

SOUSA, C. L.; CAMPOS, G. D. Condições higiênico-sanitárias de uma dieta hospitalar. Revista Nutrição, Campinas, v. 16, n. 1, p. 127-134, 2003.

SPINELLI, M. G. N.; CALE, L. R. Avaliação de resíduos sólidos em uma unidade de alimentação e nutrição. Simbio-Logias, Botucatu, v. 2, n. 1, p. 21-30, 2009.

WATTERS, C. A. et al. Exploring patient satisfaction with foodservice through focus groups and meal rounds. J Am Dietetic Assoc., v. 103, n. 10, p. 1.347-1.349, 2003.

WRIGHT, O. R. L.; CONELLY, L. B.; CAPRA, S. Consumer evaluation of hospital foodservice quality: An empirical investigation. Int J Health Care Qual Assur., v. 19, n. 2, p. 181-194, 2006.

YABUTA, C. Y.; CARDOSO, E.; ISOSAKI, M. Dieta hipossódica: aceitação por pacientes internados em hospital especializado em cardiologia. Rev. Bras. Nutr. Clin., Porto Alegre, v. 21, n. 1, p. 33-37, 2006. 